\title{
Synthesis, evaluation and release studies of NIPA nanopolymers presumed for temperature-controlled drug delivery
}

\author{
Monika Gasztych $^{1}$ Julija Volmajer Valh ${ }^{2} \cdot$ Vanja Kokol $^{2} \cdot$ Antoni Jacek Szumny $^{3}$. \\ Agnieszka Gola ${ }^{1} \cdot$ Witold Musial ${ }^{1}$
}

Received: 1 August 2015/Accepted: 14 March 2016/Published online: 25 March 2016

(C) The Author(s) 2016. This article is published with open access at Springerlink.com

\begin{abstract}
The copolymers of $N$-isopropyl acrylamide (NIPA) have been studied extensively as drug carriers for antibacterial, cardiovascular, cytostatic, local anesthetic, and nonsteroidal anti-inflammatory agents, to deliver the required therapeutic effect. The aim of this work was to evaluate the effect of a series of newly synthesized thermosensitive polymeric NIPA derivatives on the release rate of naproxen sodium (NS) from hydrogels composed of hydroxypropyl methylcellulose (HPMC). NIPA derivatives P1-P4 were synthesized by precipitation polymerization without an emulsifier, and their structures were evaluated by NMR spectroscopy. Four formulations were prepared with NS (FP1-FP4), and rate of NS release from these systems was evaluated using a modified pharmacopeial method at 22 and $42{ }^{\circ} \mathrm{C}$. The release rate of NS followed a similar trend in all four of the formulations assessed at $22{ }^{\circ} \mathrm{C}$, which was the same as the reference formulation. However, the release rate of NS at $42{ }^{\circ} \mathrm{C}$ was clearly faster in the samples containing the thermosensitive polymers P1-P4 compared with the reference formulation. These results clearly demonstrate that the use of thermosensitive
\end{abstract}

Witold Musiał

witold.musial@umed.wroc.pl

1 Department of Physical Chemistry, Pharmaceutical Faculty, Wroclaw Medical University, Borowska 211, 50-556 Wrocław, Poland

2 Laboratory for Chemistry and Environmental Protection, Faculty of Mechanical Engineering, Institute of Engineering Materials and Design, University of Maribor, Smetanova 17, 2000 Maribor, Slovenia

3 Department of Chemistry, Faculty of Food Science, Wroclaw University of Environmental and Life Sciences, ul. C. K. Norwida 25, 50-375 Wrocław, Poland polymeric derivatives of NIPA can increase the release rate of NS from hydrophilic gels based on HPMC.

Graphical Abstract Diversity of release courses of naproxen sodium evaluated in formulation containing poly(ethylene glycol) dimethacrylate (PDA, left), and comparison of half-release times ( $\mathrm{T}_{\mathrm{HR}}$, right) of naproxen sodium formulations containing various thermosensitive polymers $\mathrm{P} 1-\mathrm{P} 4$.
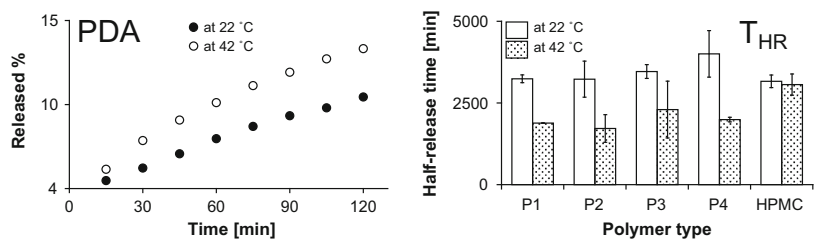

Keywords $N$-Isopropyl acrylamide - Naproxen sodium · Drug release · Hydrogel · Hydroxypropyl methyl cellulose

\section{Introduction}

Research toward the development of targeted and controlled drug delivery systems capable of carrying therapeutic agents to their required site of action has grown considerably during the last decade, because these systems offer a potential reduction in off-target effects. A variety of different methods have been evaluated to date for the preparation of specific polymeric drug carriers, including both bottom-down and bottom-up methods such as surfactant-free precipitation polymerization, surfactant-free emulsion polymerization and electrospinning [1, 2]. $\mathrm{N}$-Isopropyl acrylamide (NIPA) is an interesting monomer 
that has been applied extensively as a research tool for the development of new polymeric drug carriers [3]. The popularity of this material in scientific research can be attributed to the unique features of poly-NIPA (pNIPA) particles, which exhibit a sharp and reversible volume phase transition at a volume phase transition temperature (VPTT) of $32{ }^{\circ} \mathrm{C}$ [4-6]. The temperature of this process is especially important because it corresponds well with the range of normal human and animal body temperatures $[7$, 8]. At temperatures below the VPTT, these macromolecules become swollen when they are mixed with water. In contrast, at temperatures above their VPTT, these particles become hydrophobic and form compact structure, resulting in the dehydration of the material $[9,10]$. The synthesis of polymeric NIPA derivatives with comonomers uses an initiator, and the particles prepared in this way may be stabilized by sulfate groups derived from the persulfate ion of the attached initiator molecule [11]. It is noteworthy that a wide variety of structures may be formed during the synthesis of pNIPA macromolecules [12]. A diverse range of comonomers has been applied to the synthesis of NIPA derivatives, including acrylic acid [13], methacrylic acid [14], fumaric acid [14], acryl amide [15], maleic acid [16] and $N$-t-butyl acryl amide [17, 18]. The copolymers of NIPA have been studied extensively as drug carriers for antibacterial, cardiovascular, cytostatic, local anesthetic and nonsteroidal anti-inflammatory agents, to obtain the required therapeutic effect [19]. Although NIPA derivatives are widely recognized as potential drug carriers and nanocarriers, their practical application has been limited by their poor biodegradability. NIPA derivatives have been used effectively in the field of topical medicine, as exemplified by their use in several other studies [20-22].

Polymer structures can be readily determined nowadays using a variety of different physicochemical methods, such as infrared spectroscopy [23], mass spectrometry [24, 25], capillary electrophoresis [26] and liquid chromatography [27]. Furthermore, considerable progress has been made during the last decade toward the development of nuclear magnetic resonance (NMR) techniques for the analysis of natural [28] and synthetic polymers [29]. NMR spectroscopy represents a simple and relatively low-cost analytical technique for obtaining unambiguous structural information, making it a basic tool in polymer analysis. We previously used NMR to successfully confirm the esterification of a series of natural polymers [30-32], as well as the structure of polyNIPA microspheres [33].

The aim of the work was to evaluate the influence of our newly synthesized thermosensitive polymeric NIPA derivatives on the release rate of naproxen sodium from hydrogels composed of hydroxypropyl methylcellulose.

\section{Experiment}

\subsection{Materials}

Naproxen sodium (NS, conforming to USP standards, Sigma-Aldrich (Sternheim, Germany)), Hydroxypropylmethyl cellulose (HPMC, viscosity $2600-5600 \mathrm{cP}, 2 \%$ in $\mathrm{H}_{2} \mathrm{O}$ at $20{ }^{\circ} \mathrm{C}$, Ashland, Wilmington, DE, USA), $\mathrm{N}$-isopropyl acrylamide (NIPA, $97 \%$, Sigma-Aldrich), $N$-t-butyl acrylamide (NTB, $99 \%$, Acros organics, Geel, Belgium), $\mathrm{N}, \mathrm{N}$-methylene bisacrylamide (MBA, $99 \%$, SigmaAldrich), poly(ethylene glycol) methyl ether-acrylate with nine oxyethylene groups (PEG-MA, Mw $=480 \mathrm{Da}, 99 \%$, Sigma-Aldrich), poly(ethylene glycol) dimethacrylate (PEG-DMA, Mw $=2000 \mathrm{Da}$, Sigma-Aldrich) and potassium persulfate (KPS, $98 \%$, Sigma-Aldrich) were obtained from commercial and industrial suppliers and used without further purification. A dialysis bag with a molecular mass cutoff (MWCO) of 12,000-14,000 Da was obtained from Visking Medicell International Ltd. (London, UK). The deionized water used in the current study was obtained from an ionic column and conformed to the monography of the purified water described in the European Pharmacopoeia. Deutered dimethylsulfoxide (DMSO- $\mathrm{d}_{6}$ ) for NMR spectrometry was obtained from Euriso-Top (St Aubin, France).

\subsection{Synthesis and evaluation of polymers}

The NIPA derivatives used in the current study were synthesized by precipitation polymerization without an emulsifier (i.e., surfactant-free precipitation polymerization, SFPP). Briefly, the reactor was filled with $600 \mathrm{ml}$ of deionized water, which was heated at $70{ }^{\circ} \mathrm{C}$. The reactor was then treated with a solution of $0.5 \mathrm{~g}$ of the free radical initiator KPS in $200 \mathrm{ml}$ of deionized water. The resulting mixture was then stabilized at $70{ }^{\circ} \mathrm{C}$ before being treated with a mixture of NIPA, cross-linker and comonomer in $200 \mathrm{ml}$ of deionized water. The substrate compositions are shown in Table 1.

The particles were then purified by dialysis against deionized water until the conductivity was less than $1 \mu \mathrm{S} \mathrm{cm}^{-1}$. After 21 days, the purification procedure was complete and the samples were lyophilized using a Steris LYOVAC GT2 system (STERIS GmbH, Huerth, Germany) with vacuum values in the range of $10-100 \mathrm{~Pa}$ for $32 \mathrm{~h}$. The results of dry mass analysis experiments revealed that the concentration of the polymers in the resulting aqueous dispersions was of the order of $0.5 \%(\mathrm{w} / \mathrm{w})$.

The NMR spectra of the monomers used in the current study and the resulting polymers were measured on a 300-MHz Bruker NMR spectrometer (Bruker, City, 
Country) in the Integrated Education and Innovation Centre of the Faculty of Pharmacy at the Medical University in Wroclaw, Poland. The progress of each reaction and the chemical structures of the products were determined by ${ }^{1} \mathrm{H}$ NMR spectroscopy, as well as the use of several correlative spectra. All of the NMR experiments were performed at $24{ }^{\circ} \mathrm{C}$ using approximately $5 \mathrm{mg}$ of dry polymer in $0.8 \mathrm{ml}$ of $\mathrm{DMSO}^{-\mathrm{d}_{6}}$ (Sigma-Aldrich). All of the samples were highly soluble in DMSO- $\mathrm{d}_{6}$, which avoided the need for filtration or centrifugation.

The hydrodynamic diameters $\left(D_{\mathrm{H}}\right)$ of the newly synthesized polymers were measured using a Zetasizer Nano device (Malvern Instruments, Malvern, UK) at a wavelength of $678 \mathrm{~nm}$ in an aqueous dispersion by dynamic light scattering (DLS). The samples were diluted tenfold with deionized water and filtered through a 0.2- $\mu \mathrm{m}$ PVDF Whatman nanofilter. The filtered samples were assessed with a $173^{\circ}$ backscatter measurement arrangement using the Mark-Houwink parameters. Every measurement was multiplied five times and evaluated using version 5.03 of the Zetasizer Nano software.

\subsection{Preparation of hydrogels with naproxen sodium}

The form different hydrogels' formulations containing NS (PF1-PF4) were prepared according to the following procedure. NS $(4.0 \mathrm{~g})$ and one of the synthesized polymers ( $\mathrm{P} 1-\mathrm{P} 4,0.5 \mathrm{~g})$ were dissolved in deionized water. HPMC $(0.5 \mathrm{~g})$ was added to the resulting solution, and the mixture

Table 1 Substrate compositions of the polymers under evaluation

\begin{tabular}{lllll}
\hline Substrates $\left(\%_{\mathrm{w} / \mathrm{w}}\right)$ & \multicolumn{4}{l}{ Type of polymer } \\
\cline { 2 - 5 } & $\mathrm{P} 1$ & $\mathrm{P} 2$ & $\mathrm{P} 3$ & $\mathrm{P} 4$ \\
\hline $\begin{array}{l}\text { Type of component } \\
\text { Main monomer }\end{array}$ & & & & \\
$\quad$ NIPA & 0.50 & 0.50 & 0.50 & 0.50 \\
Cross-linker & & & & \\
$\quad \begin{array}{l}\text { MBA } \\
\text { PEG-DMA }\end{array}$ & 0.05 & 0.05 & 0.05 & - \\
$\begin{array}{l}\text { Comonomer } \\
\text { PEG-Ma }\end{array}$ & - & - & - & 0.05 \\
$\quad$ NTB & - & 0.05 & - & - \\
$\begin{array}{l}\text { Anionic initiator } \\
\text { KPS }\end{array}$ & - & - & 0.05 & - \\
Solvent \\
$\quad$ Water
\end{tabular}

NIPA $N$-isopropyl acrylamide, MBA N,N-methylene bisacrylamide, $P E G-D M A$ poly(ethylene glycol) dimethacrylate, $P E G-M A$ poly(ethyleneglycol) methyl ether-acrylate, NTB $N$-t -butyl acrylamide, KPS potassium persulfate was homogenized. The mixtures were then incubated at $25{ }^{\circ} \mathrm{C}$ for $24 \mathrm{~h}$ and used for the release experiments at various temperatures. The compositions of the different hydrogels are shown in Table 2.

\subsection{Assessment of release rates of the drug from thermosensitive polymeric preparations}

In vitro drug release experiments were performed using a dissolution tester (Erweka, Heusenstamm, Germany) to evaluate the release profiles of NS from the different materials. These experiments were conducted using a modified version of the pharmacopeial paddle dissolution method [34] at a rotation speed of $50 \mathrm{rpm}$ in $1000 \mathrm{ml}$ of deionized water. The temperature was controlled at $22 \pm 0.5$ or $42 \pm 0.5^{\circ} \mathrm{C}$ for $2 \mathrm{~h}$. Each run was carried out using two samples, with one sample in each dissolution vessel. Small samples $(1.5 \mathrm{ml})$ were withdrawn at predetermined time intervals without any replenishment to evaluate the rate of drug release. The concentration of released drug was determined spectrophotometrically by reading the absorbance of the sample at a wavelength of $298 \mathrm{~nm}$ using a UV-Vis spectrophotometer (Jasco V-530, City, Japan).

Following mathematical models, we applied zero-, firstand second-order rate equations, as well as the Higuchi equation, to evaluate the kinetics of the drug release process from the thermally controlled hydrogels. The data obtained from the in vitro drug release profiles were subsequently used to calculate the correlation coefficients $\left(r^{2}\right)$, half-release times and release rate constants $[35,36]$.

\section{Results and discussion}

\subsection{Results}

\subsubsection{Structural evaluation of the newly synthesized polymers}

The polymerization reactions conducted in the current study were readily monitored by ${ }^{1} \mathrm{H}$ NMR spectroscopy. In all cases, the vinyl protons belonging to the substrates, which appeared as multiplets in the region of 5.5-6.4 ppm, disappeared completely during the course of the polymerization reaction. This observation provided unequivocal proof that there were no monomer, oligomer or comonomer units in the product. Furthermore, the ${ }^{1} \mathrm{H}$ NMR spectra of all the polymer products contained signals in the region of 3.75-3.9 ppm, which were attributed to the methyl groups of the isopropyl groups. The corresponding methyl groups gave broad signals in the region of $1.12-1.35 \mathrm{ppm}$, while 
Table 2 Compositions of formulations FP1-FP4 with the newly synthesized polymers, and the reference formulation

\begin{tabular}{llllllll}
\hline Formulation & \multicolumn{6}{l}{ Composition } \\
\cline { 2 - 7 } & NS $(\%)$ & P1 $(\%)$ & P2 (\%) & P3 (\%) & P4 (\%) & HPMC (\%) & AQ (\%) \\
\hline FP1 & 4.0 & 0.5 & - & - & - & 0.5 & 95.0 \\
FP2 & 4.0 & - & 0.5 & - & - & 0.5 & 95.0 \\
FP3 & 4.0 & - & - & 0.5 & - & 0.5 & 95.0 \\
FP4 & 4.0 & - & - & - & 0.5 & 0.5 & 95.0 \\
REF & 4.0 & - & - & - & - & 0.5 & 95.5 \\
\hline
\end{tabular}

NS naproxen sodium; $P 1-4$ - these polymers were synthesized by SFPP using the compositions presented in Table 1; HPMC hydroxypropyl methylcellulose; $A Q$ water; $R E F$ reference formulation the ethylene groups were visible as two broad signals around 1.5 and $2.0 \mathrm{ppm}$.

The $D_{\mathrm{H}}$ values for the different polymers, as well as their VPTT values, are shown in Table 2 . The $D_{\mathrm{H}}$ values of polymers $\mathrm{P} 1-\mathrm{P} 3$ decreased when the temperature was increased from 18 to $42{ }^{\circ} \mathrm{C}$. However, the VPTT value P2 was barely detectable under the conditions of the experiment. In contrast to the other polymers, P4 showed an increase in its $D_{\mathrm{H}}$ value when the temperature was increased from 18 to $42{ }^{\circ} \mathrm{C}$. However, in a similar manner to P2, the VPTT was barely visible, as shown in Table 3.

\subsubsection{Release rates of NS from the polymeric formulations}

All four of the formulations prepared in the current study showed similar NS release profiles when they were monitored at $22{ }^{\circ} \mathrm{C}$. Furthermore, the NS release profiles of all four formulations were similar to that of the reference formulation (Figs. 1, 2a). However, when the temperature of these experiments was increased to $42{ }^{\circ} \mathrm{C}$, the rate of release increased considerably in the samples containing the thermosensitive polymers P1-P4. Notably, increasing the temperature had a much smaller impact on the rate of release from the reference preparation, indicating that this effect was caused by the thermosensitive polymers (Fig. 2a).

The details of the differences between formulations FP1-FP4 at various temperatures are shown in Tables 4 and 5 , together with the release rate constants for selected kinetic models. The regression coefficients for the different kinetic models are also shown in these tables. The results show that the Higuchi model gave the best fit in most cases.

The half-release times determined for the different formulation experiments at 22 and at $42{ }^{\circ} \mathrm{C}$ indicated that the thermosensitive polymers were having a pronounced influence on the release of NS from the polymeric hydrogel (Fig. 2b).

\subsection{Discussion}

The NMR spectra of the different polymers produced in the current study were in agreement with data published by several other authors $[37,38]$. The presence of an MBA moiety in polymers P1-P3 was confirmed by the presence of broad weak signals corresponding to the methylidene protons around $4.4 \mathrm{ppm}$, which were similar to those of the substrate and chemical shifts published by Zhang et al. [39]. The PEG-DMA and PEG-DMA cross-linker/comonomer structures in the polymers were observed as broad ethylene $\left(-\mathrm{OCH}_{2}-\mathrm{CH}_{2} \mathrm{O}-\right)_{n}$ signals around $3.52 \mathrm{ppm}$, as described previously [40]. Finally, the NTB moieties in the products were confirmed based on the presence of a narrow signal corresponding to the methyl protons of the $t$-butyl group [41]. A sample spectrum of polymer P4 is shown in Fig. 3.

The release profiles shown in Figs. 1a-d and 2a for the different formulations clearly indicate that the release process was rather complicated. The extrapolation of the release data to the $y$-axis resulted in a $y$-intercept greater than zero in all cases, which suggested that some of the
Table 3 Hydrodynamic diameter $\left(D_{\mathrm{H}}\right)$ and volume phase transition temperature (VPTT) values of the synthesized polymers

\begin{tabular}{llllll}
\hline Polymer & $D_{\mathrm{H}}$ at $18{ }^{\circ} \mathrm{C}(\mathrm{nm})$ & $\mathrm{SD}$ & $D_{\mathrm{H}}$ at $42{ }^{\circ} \mathrm{C}(\mathrm{nm})$ & $\mathrm{SD}$ & VPTT $\left({ }^{\circ} \mathrm{C}\right)$ \\
\hline P1 & 525.0 & 12.2 & 265.7 & 1.3 & 35 \\
P2 & 838.2 & 39.6 & 340.5 & 2.2 & a \\
P3 & 859.4 & 15.3 & 270.9 & 1.6 & 28 \\
P4 & 101.5 & 5.7 & 185.3 & 0.4 & a \\
\hline
\end{tabular}

P1-P4-polymers were synthesized by SFPP using the compositions shown in Table 1; SD standard deviation

a No discernible changes were observed in the $D_{\mathrm{H}}$ value of the polymer in the assessed range 

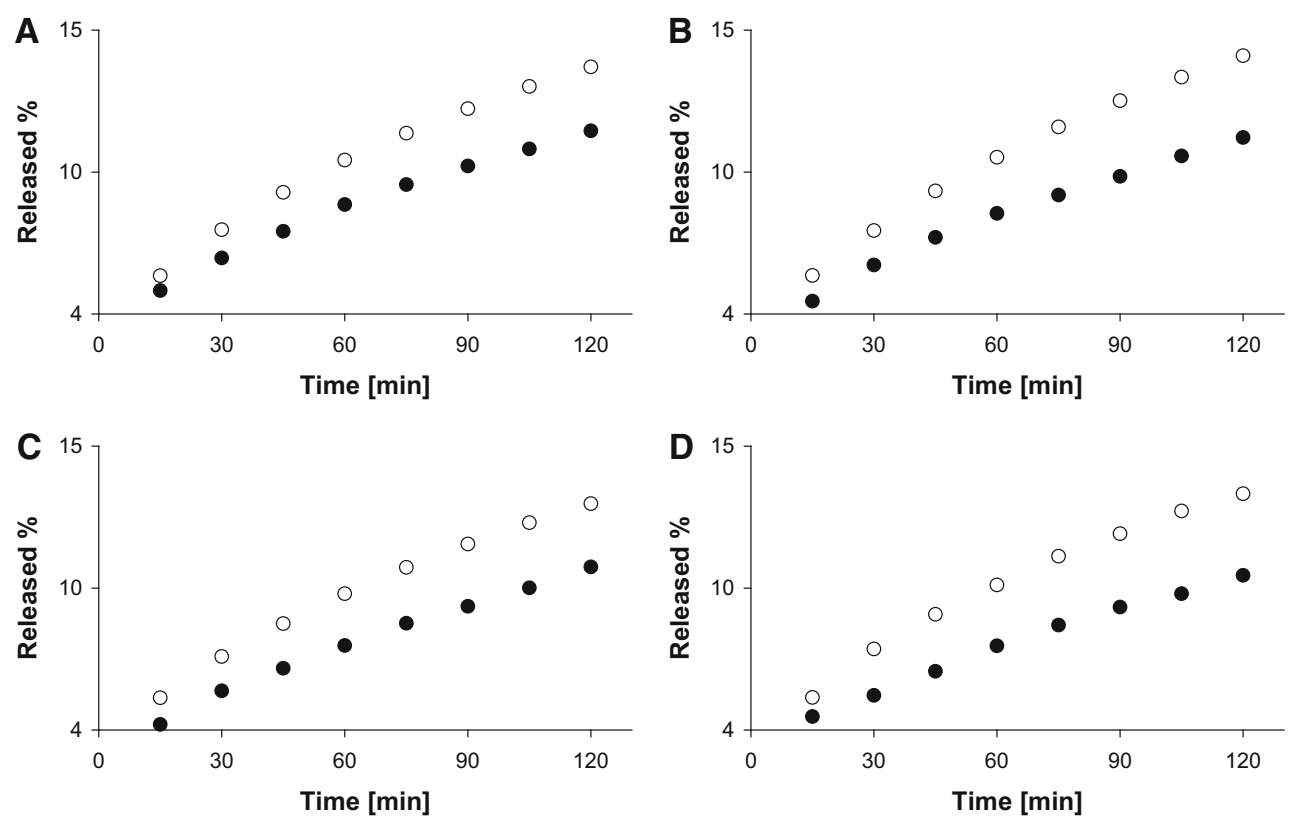

Fig. 1 Cumulative amounts of NS released from the formulations containing the thermosensitive polymers a FP1, b FP2, c FP3 and d FP4 at $22{ }^{\circ} \mathrm{C}$ (filled circle) and $42{ }^{\circ} \mathrm{C}$ (open circle)

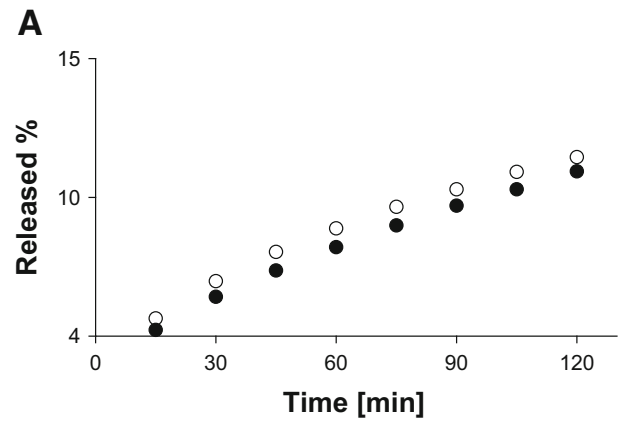

Fig. 2 a Cumulative amounts of NS released from the reference formulation containing HPMC and NS at 22 (filled circle) and $42{ }^{\circ} \mathrm{C}$ (open circle). b Half-release times $\left(t_{0.5}\right)$ for the NS released from the formulations containing the thermosensitive polymers (FP1-FP4) and

drug was present in the initial dose. This result therefore indicated that the drug had been immediately released from the formulation and that it had not bonded ironically to the polymeric structure. A similar result to this has been reported previously for polysorbate micelles containing hydrocortisone [42]. The results of the kinetic calculations revealed that the Higuchi model provided the best model for the release of NS from the different formulations. Although this model has been used in numerous studies concerning the release of drugs from polymeric matrices [43], several other models may also be used to better reflect the diffusion of drug molecules from the donor to the acceptor compartment during the release experiments [44]. The regression coefficients obtained in the current study

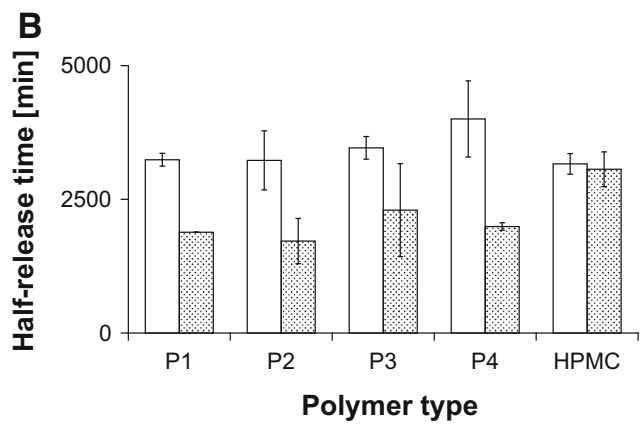

the reference formulation (HPMC) at 22 (unfilled columns) and $42{ }^{\circ} \mathrm{C}$ (filled columns). The error bars represent the standard deviations from three independent experiments

indicated that the Higuchi model provided a good fit to the experimental release patterns. The only exception to this trend was observed in the case of formulation FP4, which contained the lipophilic NIPA derivative P4 (i.e., poly-coNIPA-co-NTB). The lipophilic modification of this NIPA polymer could have an adverse influence on the release of some therapeutic agents, in a similar manner to that reported for bovine albumin [45]. It is noteworthy that the release patterns adhered more closely to the Higuchi model when the temperature of the release experiments was increased from 22 to $42{ }^{\circ} \mathrm{C}$.

The enhanced release rate of NS from the formulations prepared in the current study at $42{ }^{\circ} \mathrm{C}$, compared with the reference formulation, was attributed to the presence of the 

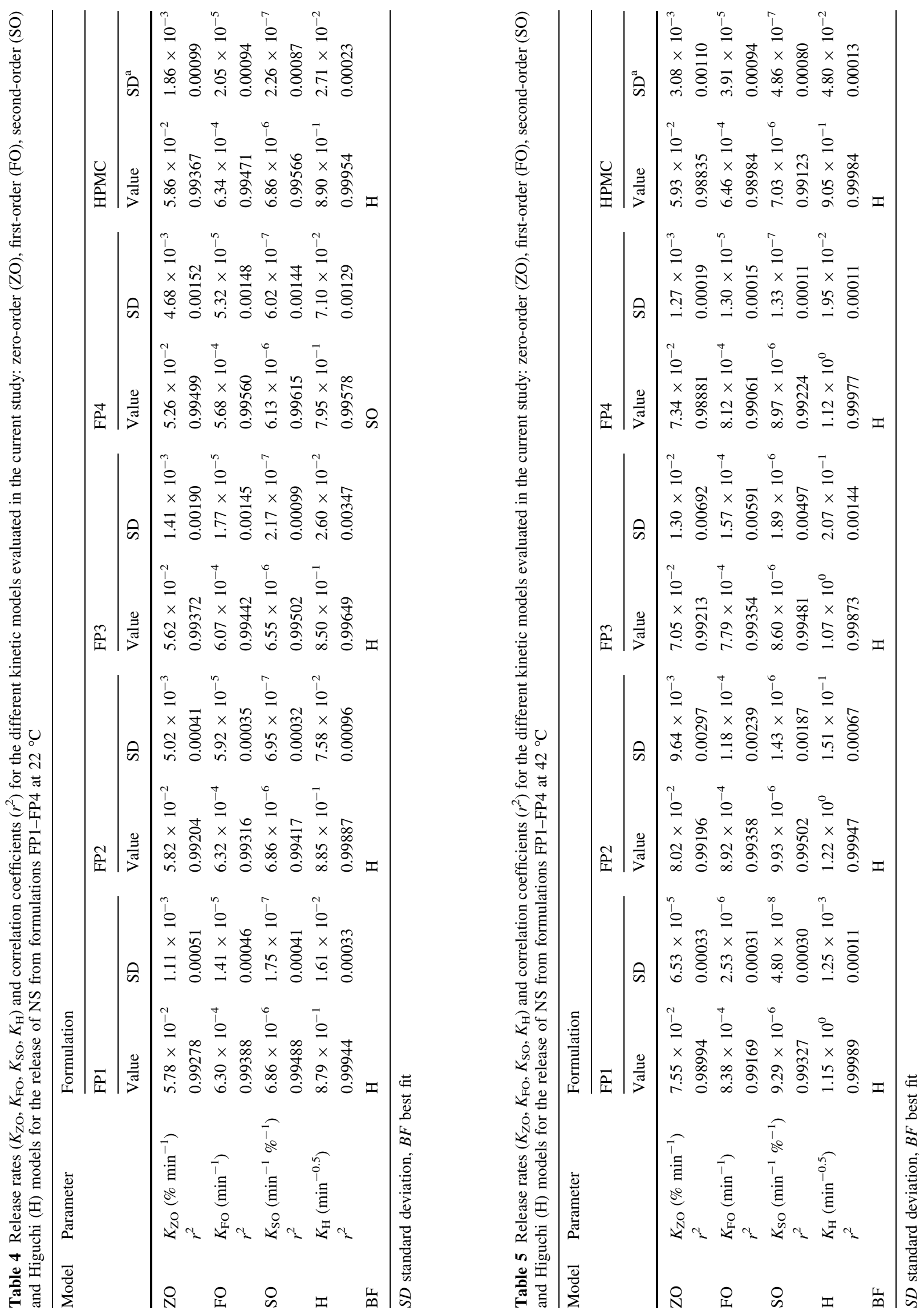


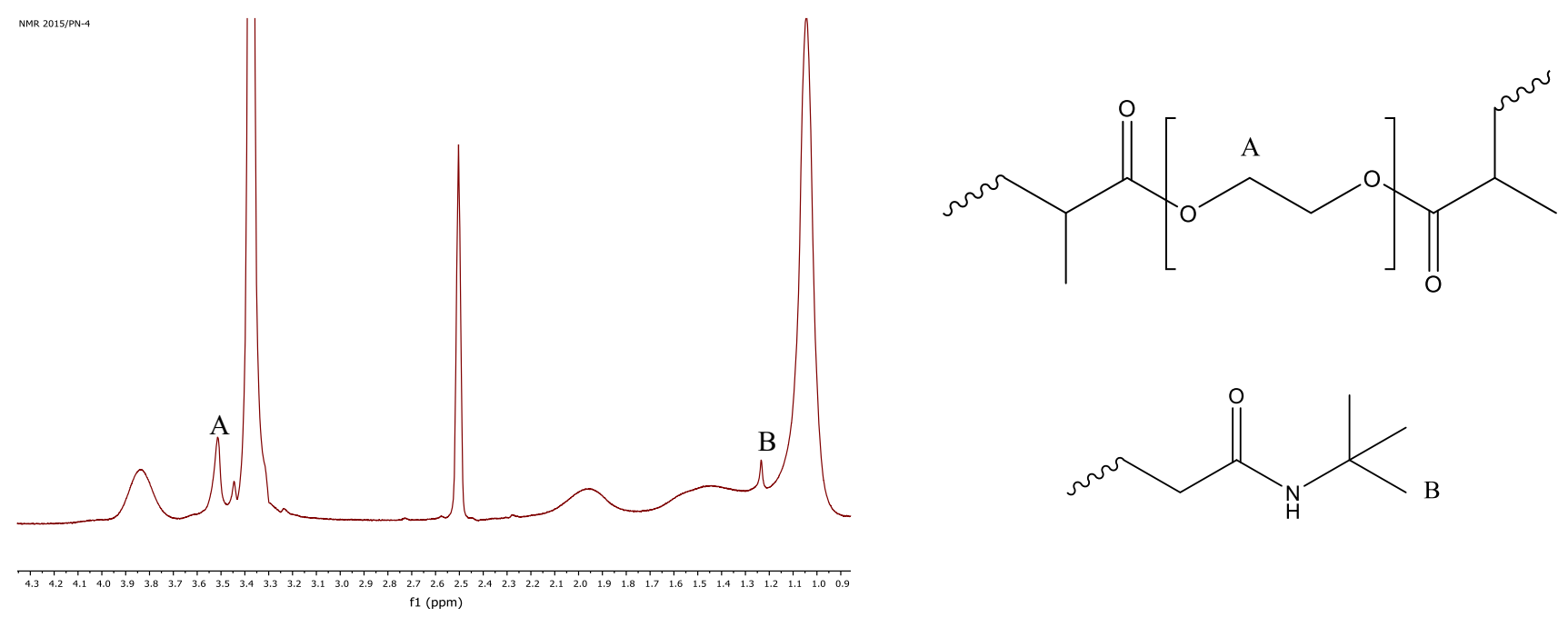

Fig. 3 NMR spectrum of P4, clearly showing the PEG-DMA cross-linker (a) and NTB comonomer (b) signals

thermosensitive polymers. This result was in agreement with those of several other reports by different authors pertaining to the use of NIPA derivatives [46, 47]. It is noteworthy, however, that this study represents the first reported account of the direct implementation of NIPA derivatives in a hydrophilic gel composed of nonthermosensitive polymers with NS.

The differences between the four different formulations FP1-FP4 were found to be negligible, which indicated that the functional groups were having very little impact on their release patterns. Formulation FP2, which contained a highly hydrophilic copolymer of NIPA and PEG-MA, behaved in a similar manner to the classic copolymer FP1 containing NIPA and MBA (FP1). In formulation FP3, the introduction of the lipophilic copolymer NTB resulted in a slight increase in the release rate of NS from the polymeric matrix to the aqueous acceptor fluid at 22 and $42{ }^{\circ} \mathrm{C}$. Although the introduction of the cross-linker PEG-DMA in FP4 led to a considerable increase in the release rate of NS, this was only observed at $22{ }^{\circ} \mathrm{C}$. The biggest difference in the release rates of NS between 22 and $42{ }^{\circ} \mathrm{C}$ was observed in the case of FP4, where the NIPA derivative was synthesized in the presence of PEG-DMA.

The modified release rates in the formulations containing the NIPA derivatives may be attributed to the specific VPTT values of the newly synthesized polymers. At temperatures above the VPTT, the structure of the polymer collapsed [48, 49], with the potential release of the NS in a similar manner to that proposed by $\mathrm{Gao}$ and $\mathrm{Hu}$ [50]. In contrast, the thermosensitive polymer can modify the structure of the HPMC hydrogel, leading to an increase in the diffusion of NS through the polymeric matrix at temperatures over the VPTT. HPMC was selected from a wide range of additives that have been previously used for the formulation of topical drugs. HPMC has an advantage over

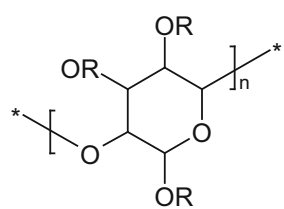

$$
\mathrm{R}=\mathrm{H} \text {, or } \mathrm{CH}_{3} \text {, or } \mathrm{CH}_{2} \mathrm{CH}(\mathrm{OH}) \mathrm{CH}_{3}
$$

Fig. 4 Structure of hydroxypropyl methyl cellulose

methyl cellulose in the sense that it can form highly transparent hydrogels [51, 52]. Furthermore, HPMC is characterized by favorable compatibility with NS and has been used as a carrier in sustained release matrix tablets [53]. Lastly, HPMC is a cellulose derivative with both hydrophilic and hydrophobic functional groups, as shown in Fig. 4.

The VPTT observed for the dispersion of pNIPA particles was attributed to the formation of hydrophobic and hydrophilic interactions between the different molecules dispersed in the water [4]. Furthermore, HPMC particles bearing a variety of different functional groups could affect the collapse of the pNIPA structure. These results do therefore not eliminate the possibility that NIPA derivatives could be successfully used as hydrogels. The polymers synthesized in the current study could also be used in a variety of other polymeric matrices, such as nanofibers, microfibers and xerogels [54, 55].

\section{Conclusions}

The results of this study have shown that the use of thermosensitive polymeric derivatives of NIPA led to an increase in the release rate of NS from hydrophilic gels 
based on HPMC. The use of PEG-DMA with the NIPA copolymer, in particular, led to the greatest decrease in the half-release time of NS. In contrast, NTB and PEG-MA both had very little impact on the half-release times. Notably, the release patterns of NS conformed to the Higuchi model in most cases. The thermosensitive copolymers of NIPA may be further evaluated as additives for the modification of release patterns of some pharmaceutically active ingredients from hydrophilic therapeutic gels.

Acknowledgments This work was prepared with a grant from the Wroclaw Medical University (Grant No. ST-848).

Open Access This article is distributed under the terms of the Creative Commons Attribution 4.0 International License (http://crea tivecommons.org/licenses/by/4.0/), which permits unrestricted use, distribution, and reproduction in any medium, provided you give appropriate credit to the original author(s) and the source, provide a link to the Creative Commons license, and indicate if changes were made.

\section{References}

1. Yang Q, Wang K, Nie J, Du B, Tang G (2014) Poly $(N$ vinylpyrrolidinone) microgels: preparation biocompatibility and potential application as drug carriers. Biomacromolecules 15:2285-2293

2. Townsend-Nicholson A, Jayasinghe SN (2006) Cell electrospinning: a unique biotechnique for encapsulating living organisms for generating active biological microthreads/scaffolds. Biomacromolecules 7:3364-3369

3. Qiu Y, Park K (2012) Environment-sensitive hydrogels for drug delivery. Adv Drug Deliv Rev 64(SUPPL):49-60

4. Pelton R (2000) Temperature-sensitive aqueous microgels. Adv Colloid Interface Sci 85:1-33

5. Kawaguchi H (2002) In: Chiellini E, Sunamoto J, Migliaresi C, Ottenbrite RM, Cohn D (eds) Biomedical functions and biotechnology of natural and artificial polymers, 1st edn. Kluwer Academic Publishers, New York

6. Cheng H, Shen L, Wu C (2006) LLS and FTIR studies on the hysteresis in association and dissociation of $\operatorname{poly}(\mathrm{N}$-isopropylacrylamide) chains in water. Macromolecules 39:2325-2329

7. Nowakowska M, Szczubiałka K, Grębosz M (2004) Modifying the thermosensitivity of copolymers of sodium styrene sulfonate and $N$-isopropylacrylamide with dodecyltrimethylammonium chloride. Colloid Polym Sci 283:291-298

8. Schwarz FP (1977) Determination of temperature dependence of solubilities of polycyclic aromatic hydrocarbons in aqueous solutions by a fluorescence method. J Chem Eng Data 22:273-277

9. Takei YG, Aoki T, Sanui K, Ogata N, Sakurai Y, Okano T (1994) Dynamic contact angle measurement of temperature-responsive surface properties for poly( $N$-isopropylacrylamide) grafted surfaces. Macromolecules 27:6163-6166

10. Plunkett KN, Zhu X, Moore JS, Leckband DE (2006) PNIPAM chain collapse depends on the molecular weight and grafting density. Langmuir 22:4259-4266

11. Odian G (2004) Principles of polymerization. Wiley, New Jersey

12. Saunders BR (2004) On the structure of poly $(N$-isopropylacrylamide) microgel particles. Langmuir 20:3925-3932
13. Zhou J, Wang G, Zou L, Tang L, Marquez M, Hu Z (2008) Viscoelastic behavior and in vivo release study of microgel dispersions with inverse thermoreversible gelation. Biomacromolecules 9:142-148

14. Hoare T, Pelton R (2008) Impact of microgel morphology on functionalized microgel-drug interactions. Langmuir 24:1005-1012

15. Hoare T, Pelton R (2008) Charge-switching amphoteric glucoseresponsive microgels with physiological swelling activity. Biomacromolecules 9:733-740

16. Das M, Sanson N, Fava D, Kumacheva E (2007) Microgels loaded with gold nanorods: photothermally triggered volume transitions under physiological conditions. Langmuir 23:196-201

17. Musial W, Vincent B, Szumny A, Voncina B (2010) Morphological characteristics of modified freeze-dried poly $(N$-isopropylacrylamide) microspheres studied by optical microscopy SEM and DLS. Chem Pap 64:602-612

18. Naha PC, Casey A, Tenuta T, Lynch I, Dawson KA, Byrne HJ, Davoren M (2009) Preparation characterization of NIPAM and NIPAM/BAM copolymer nanoparticles and their acute toxicity testing using an aquatic test battery. Aquat Toxicol 92:146-154

19. Musiał W, Pluta J, Michalek J (2015) Thermosensitive microgels of poly- $N$-isopropylacrylamide for drug carriers-practical approach to synthesis. Acta Pol Pharm 72:409-422

20. Hsiue GH, Hsu S, Yang CC, Lee SH, Yang IK (2002) Preparation of controlled release ophthalmic drops for glaucoma therapy using thermosensitive poly- $N$-isopropylacrylamide. Biomaterials 23:457-462

21. Singka GSL, Samah NA, Zulfakar MH, Yurdasiper A, Heard CM (2010) Enhanced topical delivery and anti-inflammatory activity of methotrexate from an activated nanogel. Eur J Pharm Biopharm 76:275-281

22. Musial W, Voncina B, Pluta J, Kokol V (2012) The study of release of chlorhexidine from preparations with modified thermosensitive poly- $N$-isopropylacrylamide microspheres. Sci World J 2012: 243707. doi: $10.1100 / 2012 / 243707$

23. Ren PF, Fang Y, Wan LS, Ye XY, Xu ZK (2015) Surface modification of polypropylene microfiltration membrane by grafting poly(sulfobetaine methacrylate) and poly(ethylene glycol): oxidative stability and antifouling capability. J Membr Sci 492:249-256

24. Rizzarelli P, Carroccio S (2014) Modern mass spectrometry in the characterization and degradation of biodegradable polymers. Anal Chim Acta 808:18-43

25. Altuntaş E, Schubert US (2014) "Polymeromics": mass spectrometry based strategies in polymer science toward complete sequencing approaches: a review. Anal Chim Acta 808:56-69

26. Iacob BC, Bodoki E, Oprean R (2014) Recent advances in capillary electrochromatography using molecularly imprinted polymers. Electrophoresis 35:2722-2732

27. Uliyanchenko E, van der Wal S, Schoenmakers PJ (2012) Challenges in polymer analysis by liquid chromatography. Polym Chem 3:2313-2335

28. Cheng HN, Neiss TG (2012) Solution NMR spectroscopy of food polysaccharides. Polym Rev 52:81-114

29. Todd MA, Jenkins JE, Seitz ME, Buitrago CF, Winey KI, Opper KL, Baughman TW, Wagener KB (2011) In: Cheng HN, Asakura $\mathrm{T}$, English AD (eds) NMR spectroscopy of polymers: innovative strategies for complex macromolecules, 1st edn. American Chemical Society, Washington

30. Zięba T, Kapelko M, Szumny A (2013) Effect of preparation method on the properties of potato starch acetates with an equal degree of substitution. Carbohydr Polym 94:193-198

31. Zięba T, Szumny A, Kapelko M (2011) Effect of the method for manufacturing of starch acetate on its amylolysis. Przem Chem 90:470-474 
32. Zięba T, Szumny A, Kapelko M (2011) Properties of retrograded and acetylated starch preparations: part 1 structure susceptibility to amylase and pasting characteristics. LWT-Food Sci Technol 44:1314-1320

33. Musial W, Vincent B, Szumny A, Voncina B (2010) Morphological characteristics of modified freeze-dried poly $(N$-isopropylacrylamide) microspheres studied by optical microscopy SEM and DLS. Chem Pap 64:602-612

34. The United States Pharmacopoeial Convention Inc (2007) The United States Pharmacopoeia 31st Ed-The National Formulary 26th Ed Rockville, MD

35. Dash S, Murthy PN, Nath L, Chowdhury P (2010) Kinetic modeling on drug release from controlled drug delivery systems. Acta Pol Pharm 67:217-223

36. Siepmann J, Peppas NA (2001) Modeling of drug release from delivery systems based on hydroxypropyl methylcellulose (HPMC). Adv Drug Deliv Rev 48:139-157

37. Tokuhiro T, Amiya T, Mamada A, Tanaka T (1991) NMR study of poly( $N$-isopropylacrylamide) gels near phase transition. Macromolecules 24:2936-2943

38. Sun P, Li B, Wang Y, Ma J, Ding D, He B (2003) ${ }^{1} \mathrm{H}$ NMR studies of poly $(N$-isopropylacrylamide) gels near the phase transition. Eur Polym J 39:1045-1050

39. Zhang Y, Huang W, Zhou Y, Yan D (2007) A physical gel made from hyperbranched polymer gelator. Chem Commun 25:25872589

40. Woodward RT, Slater RA, Higgins S, Rannard SP, Cooper AI, Royles BJL, Weaver JVM (2009) Controlling responsive emulsion properties via polymer design. Chem Commun 24:3554-3556

41. Ibrahim K, Löfgren B, Seppälä J (2003) Synthesis of tertiarybutyl acrylate polymers and preparation of diblock copolymers using atom transfer radical polymerization. Eur Polym J 39: 2005-2010

42. Kubis AA, Musial W, Szczesniak M (2002) Influence of some polysorbates on hydrocortisone release from hydrophilic gels considered as two-compartment models. Pharmazie 57:479-481

43. Siepmann J, Peppas NA (2012) Modeling of drug release from delivery systems based on hydroxypropyl methylcellulose (HPMC). Adv Drug Deliv Rev 64(SUPPL):163-174

44. Costa P, Sousa Lobo JM (2001) Modeling and comparison of dissolution profiles. Eur J Pharm Sci 13:123-133
45. Huo D, Li Y, Qian Q, Kobayashi T (2006) Temperature-pH sensitivity of bovine serum albumin protein-microgels based on cross-linked poly( $N$-isopropylacrylamide-co-acrylic acid). Colloids Surf B 50:36-42

46. Taşdelen B, Kayaman-Apohan N, Güven O, Baysal BM (2004) Preparation of $\operatorname{poly}(N$-isopropylacrylamide/itaconic acid) copolymeric hydrogels and their drug release behavior. Int $\mathbf{J}$ Pharm 278:343-351

47. Coughlan DC, Quilty FP, Corrigan OI (2004) Effect of drug physicochemical properties on swelling/deswelling kinetics and pulsatile drug release from thermoresponsive $\operatorname{poly}(N$-isopropylacrylamide) hydrogels. J Control Release 98:97-114

48. OkadaY Tanaka F (2005) Cooperative hydration, chain collapse, and flat LCST behavior in aqueous poly ( $N$-isopropylacrylamide) solutions. Macromolecules 38:4465-4471

49. Wu C, Zhou S (1995) Laser light scattering study of the phase transition of poly $(N$-isopropylacrylamide) in water. 1. Single chain. Macromolecules 28:8381-8387

50. Gao J, Hu Z (2002) Optical properties of $N$-isopropylacrylamide microgel spheres in water. Langmuir 18:1360-1367

51. Marques-Marinho FD, Vianna-Soares CD (2013) In: van de Ven Theo, Godbout Louis (eds) Cellulose and its derivatives use in the pharmaceutical compounding practice, 1st edn. Rijeka, InTech

52. Curtis-Fisk J, Sheskey P, Balwinski K, Coppens K, Mohler C, Zhao J (2012) Effect of formulation conditions on hypromellose performance properties in films used for capsules and tablet coatings. AAPS PharmSciTech 13:1170-1178

53. Dahl TC, Calderwood T, Bormeth A, Trimble K, Piepmeier E (1990) Influence of physico-chemical properties of hydroxypropyl methylcellulose on naproxen release from sustained release matrix tablets. J Control Release 14:1-10

54. Jayasinghe SN (2013) Cell electrospinning: a novel tool for functionalising fibres, scaffolds and membranes with living cells and other advanced materials for regenerative biology and medicine. Analyst 138:2215-2223

55. Cortés JA, Mendizábal E, Katime I (2008) Effect of comonomer type and concentration on the equilibrium swelling and volume phase transition temperature of $\mathrm{N}$-isopropylacrylamide-based hydrogels. J Appl Polym Sci 108:1792-1796 